\title{
Mô hình quản lý công ty bất động sản
}

\section{Real estate company management model}

\author{
Dương Tuyết Ngọc ${ }^{1,2^{*}}$ \\ ${ }^{1}$ Trường Đại Mở Thành phố Hồ Chí Minh, Việt Nam \\ ${ }^{2}$ Ban quản lý dự án đầu tư xây dựng khu vực Quận 10, Việt Nam \\ *Tác giả liên hệ, Email: duongtuyetn@gmail.com
}

\section{THÔNG TIN}

DOI: $10.46223 /$ HCMCOUJS. proc.vi.16.1.1854.2021

Ngày nhận: 7/3/2021

Ngày nhận lại: 31/3/2021

Duyệt đăng: 7/4/2021

Tù khóa:

mô hình quản lý bất động sản doanh nghiệp, mô hình quản lý, CREM, quản lý doanh nghiệp

Keywords:

corporate real estate, corporate real estate management, CREM, real estate management, management model, facilities management

\section{TÓM TĂT}

Trong những năm vừa qua, Bất động sản doanh nghiệp (Corporate Real Estate - $\mathrm{CRE}$ ) được các nhà nghiên cứu khoa học cho là tài sản không được đánh giá cao bởi các nhà quản lý cấp cao của doanh nghiệp vì chưa được phát huy tối đa mặc dù nó chiếm chi phí khá lớn của doanh nghiệp. Nếu được quản lý phù hợp sẽ mang lại cổ tức cho hoạt động kinh doanh cốt lõi của doanh nghiệp. Thật vậy, nó rất quan trọng đối với sự thành công của một tổ chức doanh nghiệp bất động sản và cũng được biết đến là yếu tố chi phí trong quan trọng trong ngân sách doanh nghiệp. Bài báo này là một nghiên cứu phân tích tài liệu các vấn đề chung về Quản lý Bất động sản Doanh nghiệp (Corporate Real Estate Management - CREM). Nội dung bài viết nhấn mạnh việc hình thành khái niệm, hoạt động các lĩnh vực, thành phần và đưa ra các ví dụ thực tế của CREM. Kết quả nghiên cứu cho thấy một bức tranh tổng thể có hệ thống về CREM và xác định các vấn đề hiện tại mà CREM đang gặp tại các doanh nghiệp bất động sản.

\section{ABSTRACT}

In recent years, Corporate Real Estate (CRE) has been considered by scientific researchers to be an asset that is not appreciated by senior managers of the business because it has not been maximized although it accounts for a sizable cost of the business. Proper management will bring dividends for the core business of the business. Indeed, it is crucial to the success of a real estate business organization and is also known to be the key cost factor in the corporate budget. This paper is a documentary analysis study of Corporate Real Estate Management (CREM) issues. The content of the article emphasizes the conceptual formation, activities of the domains, components and gives practical examples of CREM. The research results show a systematic overview of CREM and identify the current problems that CREM is facing in real estate businesses.
\end{abstract}

\section{Giới thiệu}

Quản lý Bất động sản Doanh nghiệp (CREM), (được gọi dưới các tên sau đây: danh mục 
Bất động sản Quản lý, Quản lý tài sản bất động sản doanh nghiệp và Quản lý Tài sản Bất động sản) được coi là một lĩnh vực mới trong cộng đồng kinh doanh bất động sản. Nó thường được cho là liên quan đến việc quản lý các tòa nhà và các lô đất dưới sự quyết định của các tổ chức không chủ yếu kinh doanh bất động sản (Bon, 1995). Quản lý của các tòa nhà được tổ chức cho các mục đích hoạt động như quản lý danh mục đầu tư bất động sản trong đó mục đích chính là tối đa hóa lợi ích tài chính cho các chủ sở hữu (Scarrett, 2010). Những tài sản này về cơ bản không được sử dụng cho thu nhập thế hệ. CREM nhằm thiết lập và duy trì sự phù hợp chặt chẽ giữa chiến lược kinh doanh và tài sản của một doanh nghiệp (Bon, 1995). Nó làm tăng giá trị cho doanh nghiệp bằng cách tăng sự hài lòng của khách hàng; nâng cao hiệu quả; và cải thiện năng suất hoặc cung cấp dịch vụ bằng cách kết hợp chiến lược bất động sản vào tổng thể công ty chiến lược (Lambert \& Poteete, 1997; Scarrett, 2010).

Bất động sản là một trong những thị trường quan trọng trong nền kinh tế quốc dân vì nó liên quan đến một lượng tài sản lớn. Tài sản bất động sản thường chiếm khoảng $45-75 \%$ sự giàu có của các nước đang phát triển và ít nhất bằng $20 \%$ GDP của những nước này. Việc phát triển thị trường bất động sản có tác động đến tăng trưởng kinh tế thông qua: kích thích đầu tư vào đất đai, nhà xưởng, chuyển dịch lao động giữa các ngành, các vùng lãnh thổ thông qua đầu tư và kinh doanh bất động sản, chuyển bất động sản thành tài sản tài chính. Phát triển thị trường bất động sản trong thời gian tới là cần thiết. Trong đó, Quản lý Bất động sản Doanh nghiệp (CREM) đã nhận được sự quan tâm đáng kể ở các nước phát triển như Mỹ và Anh. Việc sử dụng mô hình CREM trong việc quản lý kinh doanh ở các doanh nghiệp lớn rất được ưu chuộng và đã mang lại kết quả tổng thể cao hơn hiệu suất lợi nhuận. Trong năm 2004, bất động sản được ước tính chiếm khoảng 20-30\% tổng tài sản ở Mỹ và 30-35\% ở Vương quốc Anh với Tây Âu dự kiến sẽ có số liệu cao hơn (Luokko, 2004; Njungbwen \& Udo, 2010). Năm 2007, người ta ước tính rằng danh mục đầu tư của chính phủ Anh bao gồm 13 diện tích sàn văn phòng triệu mét vuông, trị giá 30 tỷ bảng Anh và chi phí hàng năm là 6 tỷ bảng Anh. Báo cáo kết luận rằng việc quản lý tài sản không hiệu quả đã làm chính phủ thiệt hại hơn 326 triệu bảng mỗi năm so với mức cần thiết (Jones \& White, 2012). (Hartmann, Linneman, Pfnuer \& Siperstein, 2009) tuyên bố rằng Bất động sản doanh nghiệp (CRE) luôn là một tài sản có giá trị đối với bảng cân đối kế toán, ngay cả khi nhiều doanh nghiệp không biết đến thực tế này.

\section{Nội dung}

\subsection{Lịch sủ̉ phát triển}

Quan điểm của doanh nghiệp về quản lý bất động sản đã thay đổi kể từ những năm 1980 (Krumm, 2001). Các tòa nhà thực sự đã được biết đến trước khi bắt đầu thời đại hiện đại, nhưng cho đến thế kỷ 19 , chúng được xây dựng để tạo ấn tượng tượng trưng về nhà nước, về những đặc trưng mang tính chất của mỗi quốc gia. Kinh phí lúc đó không có nhiều vấn đề. Rất nhiều hoạt động công nghiệp, chủ yếu là quy mô nhỏ, không yêu cầu nhiều chỗ ở cho đến thời đại cuộc cách mạng công nghiệp. Thời kỳ này kêu gọi chỗ ở cho nền công nghiệp lớn hơn các hoạt động và do đó là việc quản lý các bất động sản đó. Sự xuất hiện của hai các tổ chức từ Hoa Kỳ đến Vương quốc Anh khoảng hai thập kỷ trước đã tập trung vào hoạt động quản lý tài sản hoặc CRE như một lĩnh vực hoạt động chuyên nghiệp duy nhất. Đến từ quan điểm toàn cầu trong nghiên cứu dọc của họ về CREM chỉ ra rằng, Quản lý Bất động sản Doanh nghiệp (CREM) lần đầu tiên xuất hiện trên chương trình nghiên cứu năm 1980. Vào thời điểm đó, trọng tâm của nghiên cứu là đạt được hiểu biết về cách quản lý tài nguyên bất động sản của công ty (CRE) trong phạm vi rộng tổ chức (Bon, 1995). Những các nghiên cứu tìm kiếm bằng chứng về các thực hành quản lý "tốt" và dựa trên phân tích của điều tra bảng câu hỏi, bổ sung dữ liệu phỏng vấn định tính. Sự kết luận đạt được từ các nghiên cứu này nêu bật mối quan tâm rằng $\mathrm{CRE}$ đã được quản lý và các tổ chức đã không thể 
khai thác hết giá trị hoặc tiềm năng của nó. Đầu những năm 1990 chứng kiến sự thay đổi về trọng tâm khi các nhà nghiên cứu bắt đầu kiểm tra các yếu tố của quá trình quản lý. Các chủ đề nghiên cứu bao gồm việc kiểm tra mối liên hệ giữa chiến lược của tổ chức và chiến lược $C R E$ của nó và sự phát triển của các hệ thống đo lường hiệu suất. Trong một số trường hợp các bài báo này đã cố gắng phát triển lý thuyết $\mathrm{CRE}$ có liên quan đến quản lý văn học và những kiến thức $\mathrm{CRE}$ nền tảng khác về tài liệu nghiên cứu điển hình (Bon, 1995).

Có bốn quan điểm của CREM là Quản lý chung, Quản lý tài sản, Quản lý cơ sở và Kiểm soát chi phí. Bốn điều này được sử dụng để làm nổi bật cách tiếp cận phân khu để quản lý bất động sản so với CREM tổng thể cách tiếp cận. Các chức năng quản lý của CREM cũng được chia thành các lĩnh vực chính sau: trọng tâm kinh doanh, trọng tâm hoạt động, địa ốc tiêu điểm, và trọng tâm chiến lược. Đây một lần nữa, tổ chức giải quyết các lĩnh vực này một cách riêng biệt thay vì quản lý chúng cùng nhau vì lợi ích của tổ chức nói chung. Ít nhất bốn lĩnh vực quan trọng của thực hành CRE hiện tại dưới ánh sáng quản lý một thực thể kinh doanh cụ thể là: Đo lường Hiệu quả Kinh doanh CRE; CRE Cấu trúc tổ chức; Quản lý và Đánh giá Rủi ro CRE; và CRE Strategic Lập kế hoạch. Nghiên cứu được thực hiện trong lĩnh vực $\mathrm{CRE}$ có xu hướng dựa trên số lượng dữ liệu hạn chế. Phân tích dữ liệu CRE của họ đã xác định được 32 các thành phần tạo nên các thuộc tính của chính sách, chức năng và hoạt động $\mathrm{CREM}$. Những điều quan trọng nhất tuân theo thứ tự này: phương thức làm việc mới, gia công phần mềm, cơ sở hạ tầng công nghệ và quản lý và chiến lược $\mathrm{CRE}$.

\subsection{Muc tiêu của CREM}

Mục đích của CREM là nâng cao hiệu suất của tổ chức thông qua sự liên kết chiến lược bất động sản của công ty với chiến lược tổ. Do đó, CREM cần tạo ra tác động lớn đến sức mạnh và khả năng cạnh tranh của một tổ chức bằng cách đảm bảo rằng các bất động sản được sử dụng hiệu quả. Từ tổng hợp ba nghiên cứu Musa và Baharum (2012) xác định chi phí tối thiểu, tăng giá trị, tăng tính linh hoạt và xúc tiến thị trường và bán hàng như các thành phần chung của giá trị gia tăng từ $\mathrm{CRE}$. Tuy nhiên, không chú ý đến vai trò gia tăng giá trị của CREM bằng cách hỗ trợ chiến lược cho cốt lõi kinh doanh của các tổ chức. Mục tiêu chính của CREM phải là tạo ra lợi nhuận từ bất động sản mà không thay đổi trọng tâm của tổ chức từ hoạt động kinh doanh cốt lõi của nó (Hartmann, Linneman, Pfnür, Moy, \& Siperstein, 2010). Do đó cần có các tổ chức hướng sự tập trung của họ vào quan điểm chiến lược của Bất động sản Doanh nghiệp như một công cụ quan trọng của doanh nghiệp. Điều này có thể yêu cầu trình diện các Giám đốc điều hành Bất động sản công ty yêu cầu đào tạo bởi các cơ quan chuyên môn có liên quan sẽ trang bị cho họ để hoạt động có hiệu quả. Sự đóng góp của nhân viên CRE cũng sẽ cảm thấy và được đánh giá cao hơn khi họ chuyển dần lên ở cấp chức năng đến vai trò cấp cao nhất của một nhà chiến lược kinh doanh trong đó chức năng của họ là tích hợp lực lượng lao động, nơi làm việc và xu hướng công nghệ vào chiến lược kinh doanh tổng thể.

\subsection{Nguyên tắc của CREM}

Bon (1994) đã trình bày mười nguyên tắc của CREM (Bon, 1995). Ông đã cho thấy rằng, mối quan tâm của CREM là thiết lập và duy trì sự phù hợp chặt chẽ giữa các chiến lược kinh doanh và tài sản. Nó xử lý toàn bộ chu kỳ tài sản thực trong danh mục đầu tư và quan tâm đến mối quan hệ giữa chu kỳ tài sản thực, các chu kỳ chiến lược đặc trưng khác được các doanh nghiệp đề cập. CREM cung cấp các phương pháp và công cụ hỗ trợ việc hình thành và duy trì vòng lặp phản hồi giữa hiệu suất tài sản thực trong danh mục đầu tư và hoạt động của người quản lý. Nó yêu cầu giám sát hiệu quả hoạt động của tài sản bằng một hệ thống tài sản biểu hiệu qua chỉ số hoạt động. Sự cải tiến liên tục và gia tăng phù hợp với thay đổi mục tiêu của doanh nghiệp bất động sản được xác định là lý thuyết của CREM và mối quan tâm chính của CREM là sự duy trì khả năng liên tục thích ứng với các điều kiện kinh tế thay đổi đối mặt với một doanh nghiệp. 


\subsection{Lĩnh vục hoạt động của CREM}

Tám lĩnh vực hoạt động chính của CREM như có thể thấy trong bảng sau:

\section{Bảng 1}

Cơ cấu hoạt động trong CREM (Lambert \& Poteete, 1997)

\begin{tabular}{|c|c|c|c|}
\hline \multirow{2}{*}{$\begin{array}{l}\text { Hoạt động của } \\
\text { CREM }\end{array}$} & \multicolumn{3}{|c|}{ Mức độ quan trọng } \\
\hline & Quan trọng nhất & Quan trọng thứ yếu & Ít quan trọng \\
\hline $\begin{array}{l}\text { Quản lý bất động sản, } \\
\text { tài chính và luật }\end{array}$ & Giai đoạn đầu tư & Giai đoạn quyết toán & $\begin{array}{c}\text { Giai đoạn chuẩn bị đầu } \\
\text { tư }\end{array}$ \\
\hline Mua, bán và cho thuê & Giai đoạn đầu tư & Giai đoạn quyết toán & $\begin{array}{c}\text { Giai đoạn chuẩn bị đầu } \\
\text { tư }\end{array}$ \\
\hline $\begin{array}{l}\text { Thiết kế, xây lắp, cải } \\
\text { tạo }\end{array}$ & Giai đoạn đầu tư & Giai đoạn quyết toán & $\begin{array}{c}\text { Giai đoạn chuẩn bị đầu } \\
\text { tư }\end{array}$ \\
\hline Quản lý thị trường & Giai đoạn quyết toán & $\begin{array}{c}\text { Giai đoạn chuẩn bị } \\
\text { đầu tư }\end{array}$ & Giai đoạn đầu tư \\
\hline Dịch vụ hỗ trợ & Giai đoạn quyết toán & $\begin{array}{c}\text { Giai đoạn chuẩn bị } \\
\text { đầu tư }\end{array}$ & Giai đoạn đầu tư \\
\hline $\begin{array}{l}\text { Quản lý và bảo trì dịch } \\
\text { vụ tòa nhà }\end{array}$ & Giai đoạn quyết toán & $\begin{array}{c}\text { Giai đoạn chuẩn bị } \\
\text { đầu tư }\end{array}$ & Giai đoạn đầu tư \\
\hline Quản lý tòa nhà & $\begin{array}{c}\text { Giai đoạ̀ chuẩn bị } \\
\text { đầu tư }\end{array}$ & Giai đoạn quyết toán & Giai đoạn đầu tư \\
\hline Bảo trì tòa nhà & $\begin{array}{c}\text { Giai đoạn chuẩn bị } \\
\text { đầu tư }\end{array}$ & Giai đoạn quyết toán & Giai đoạn đầu tư \\
\hline
\end{tabular}

Nguồn: Kết quả phân tích dữ liệu của nhóm nghiên cứu

Ví dụ những phân tích của các tòa nhà trong ma trận SWOT, về các yếu tố bên ngoài và các yếu tố bên trong ảnh hưởng đến kết quả của hậu công suất đánh giá (POE) và hiệu quả chi phí của các tòa nhà được thể hiện sau đây.

\section{Bảng 2}

Ma trận SWOT đánh giá các tòa nhà riêng lẻ

\begin{tabular}{|c|c|c|}
\hline \multirow{2}{*}{ Các yếu tố bên trong } & \multicolumn{2}{|c|}{ Các yếu tố bên ngoài } \\
\hline & Co' hội (Opportunities) & Mối đe dọa (Threats) \\
\hline Điểm mạnh (Strengths) & $\begin{array}{l}\text { SO - tòa nhà nên được bảo } \\
\text { tồn, cả dự án và vị trí đều } \\
\text { thỏa đáng, nó là một ví dụ về } \\
\text { xây dựng tốt }\end{array}$ & $\begin{array}{l}\text { ST - Vị trí có thể không tốt, địa điểm } \\
\text { sẽ bị bán. Dự án nên được nghiên cứu }\end{array}$ \\
\hline Điểm yếu (Weaknesses) & $\begin{array}{l}\text { WO - tòa nhà xấu ở một vị } \\
\text { trí tốt, thuận lợi cho việc sửa } \\
\text { chữa lại hoặc thay thế }\end{array}$ & $\begin{array}{l}\text { WT - cả dự án và vị trí đều thiếu, một } \\
\text { ví dụ điển hình về việc xây dựng và } \\
\text { một ứng cử viên để thoái vốn }\end{array}$ \\
\hline
\end{tabular}


Bon (1994) gần như lặp lại vị trí trên bằng cách nói rằng CREM bao gồm toàn bộ một loạt các hoạt động liên quan đến danh mục các tòa nhà và đất đai. Bao gồm các: kế hoạch và quản lý đầu tư, kế hoạch và quản lý tài chính, xây dựng lập kế hoạch và quản lý, quy hoạch và quản lý cơ sở vật chất. Các chức năng của CREM bao gồm: lập kế hoạch và thực hiện việc mua lại bất động sản và thải bỏ, quản lý không gian, quy hoạch và lắp đặt nội thất; kiến trúc và kỹ thuật các dịch vụ; bảo trì và lập ngân sách hoạt động. Nội dung và nhiệm vụ của CREM khác nhau giữa các doanh nghiệp (Njungbwen \& Udo, 2010). Tuy nhiên, mục đích quan trọng là hỗ trợ hoạt động kinh doanh cốt lõi của doanh nghiệp bất động sản. Họ nghĩ rằng thành công của CREM do cần được đo lường dựa trên khả năng tạo ra giá trị gia tăng cho doanh nghiệp và đóng góp vào kết quả hoạt động chung của doanh nghiệp do đó, đòi hỏi các nhu cầu và mục tiêu của hoạt động kinh doanh cốt lõi phải rất rõ ràng.

\subsection{Phân tích mô hình CREM}

Các lớp và lớp con được xem xét trong kích thước được thể hiện trong hình dưới đây. $\mathrm{Ba}$ mỗi lớp chính được chia thành ba các lớp con; các lớp con này cũng được chia thành một số biến của các lớp con khác. Mỗi lớp con cấp cuối cùng chứa một số lượng nhóm tính năng thay đổi được sử dụng trong CREM. Mỗi các lớp con cấp cuối cùng này có thể bao gồm nhiều các tính năng cần thiết để thực hiện các nhiệm vụ liên quan đến một khía cạnh cụ thể của quản lý bất động sản

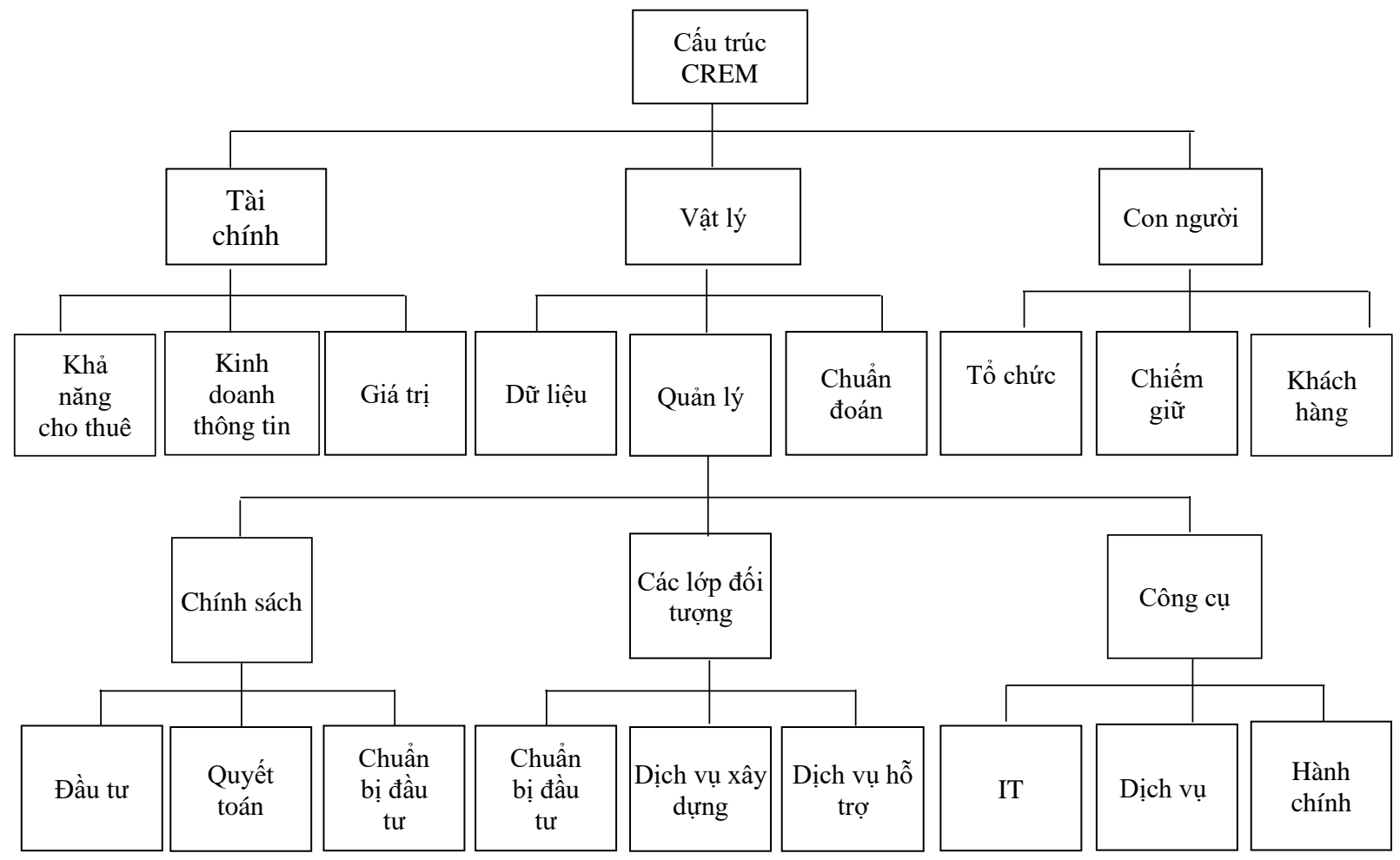

Hình 1. Cấu trúc của CREM

Mỗi lớp con cấp cuối cùng của cấu hình CREM chứa một số biến của các nhóm tính năng, từng nhóm tính năng bao gồm một nhóm các phương pháp, công cụ và các khái niệm được sử dụng trong một hoạt động cụ thể trong các hoạt động CREM. Ví dụ, chỉ lớp con cấp cuối cùng, "dữ liệu”, được mô tả định nghĩa của lớp con này là tài sản đăng ký cộng với các thanh ghi dữ liệu khác, bao gồm cả các kỹ thuật và các công cụ được sử dụng để lưu trữ dữ liệu và vận hành sổ đăng ký, dữ liệu được sử dụng cho hoạt động thay vì dữ liệu tài chính được sử dụng. Trong mô hình dữ liệu công ty, những dữ liệu sẽ là dữ liệu cục bộ để thực hiện hoạt động nhiệm vụ theo thời gian và 
dữ liệu không quan trọng trong chiến lược công ty. Lớp con này có ba nhóm tính năng:

(1) Dũ liệu: Sổ đăng ký tài sản; lịch sử; các cửa hàng, độ bền, lỗi thời và thông số kỹ thuật; dữ liệu quản trị (ví dụ: dữ liệu mua hàng, Bảo hành, địa chỉ tòa nhà, đồ nội thất, nhà cung cấp, bảo trì, cho thuê thông tin....)

(2) Kỹ thuật: Mã hóa tài sản hợp nhất cấu trúc, hệ thống mã hóa, xây dựng dòng sản phẩm.

(3) Công cụ: Gắn thẻ mã vạch, đầu đọc mã vạch, Tệp CAD (ví dụ: nhà máy, thiết bị, dịch vụ tệ nạn, đồ đạc, thư viện biểu tượng).

Một số mô hình tập trung vào các khía cạnh phần mềm (ví dụ: AutoFM, ArchibusFM), những dịch vụ khác, kiểm soát các nhiệm vụ vận hành (ví dụ: StarTrak), và chi phí, giá trị liên quan đến bất động sản (ví dụ tiền, tài chính). Những khác biệt này phụ thuộc vào sự phát triển nguồn gốc của mô hình và cả về sự tồn tại của hệ thống CAD như một phần hiệu quả của phần mềm. Ngôn ngữ hướng đối tượng (ví dụ: $\mathrm{C}++$ ) tích hợp một hệ thống $\mathrm{CAD}$ và mối quan hệ cơ sở dữ liệu, cho phép sử dụng dữ liệu vật lý thuộc tính được lưu trữ trong tệp CAD dưới dạng cơ sở của hệ thống.

Sự khác biệt này không quan trọng lắm ở cấp chiến lược của quản lý danh mục đầu tư. bên trong mức độ chiến thuật và hoạt động của các cơ sở quản lý, cơ sở $\mathrm{CAD}$ cho phép tích hợp thiết kế và xây dựng các giai đoạn với quản lý tòa nhà và giai đoạn hoạt động. Về lâu dài, hiệu quả tin tức được cung cấp bởi hệ thống CAD trong sử dụng thông tin phản hồi cho các giai đoạn quản lý chương trình và dự án, và giảm thời gian dẫn của mới triển khai, có thể tạo ra sự cạnh tranh thuận lợi cho tổ chức một nhiệm vụ cơ hội trong một lĩnh vực mới nổi nơi nó có thể giúp chính thức hóa nền tảng lý thuyết của nó đất. CREM và các môn học liên quan như quản lý cơ sở vật chất (FM) hoặc công ty thực tài sản hoặc quản lý tài sản là những lĩnh vực mà thông tin rất đa dạng và không được cấu trúc tốt kiểm tra, tiêu biểu của các đối tượng mới nổi. Nó quan trọng để phát triển lý thuyết cơ bản và tiết lộ cấu trúc. Nỗ lực phân loại các khía cạnh được xử lý bởi các nhà nghiên cứu và những người hành nghề trong lĩnh vực này trong một khuôn khổ, cũng như mô tả của các các hoạt động hình thành kỷ luật, là một sáng kiến có thể nâng cao tổ chức và sản xuất kiến thức trong chủ đề vấn đề.

Những phát triển trong tương lai của nghiên cứu này sẽ là sự bổ sung của nhiều mô hình hơn trong phân tích, ứng dụng của cụm thống kê và phân tích tương ứng với dữ liệu, với mục đích của việc tạo ra nhiều bằng chứng hơn để xây dựng mô hình. Thú vị khác sự phát triển của nghiên cứu này sẽ là ứng dụng mô hình để chẩn đoán Các đơn vị CREM trong doanh nghiệp, trường hợp tạo nghiên cứu, đó là một phương pháp thích hợp để đối phó với sự phức tạp của vấn đề.

\section{Kết luận}

Sự liên quan của bất động sản đối với tất cả các loại hình doanh nghiệp. Tương tự như vậy, sự quản lý kém của nó đã được chứng minh. Do đó, CREM tập trung vào sự chủ động quản lý chuyên nghiệp bất động sản và các cơ sở liên quan một cách tổng thể để đạt được mục tiêu của các chủ thể doanh nghiệp. Nó giao dịch với tất cả các loại tài sản: công cộng, tư nhân, bán công. Ban đầu, nó được cho là hoạt động trong các tổ chức phi bất động sản nhưng theo lý thuyết của các nhà nghiên cứu khác, nó đã đưa ra rằng nó cũng diễn ra trong các tổ chức liên quan trong kinh doanh bất động sản. Trên thực tế, điểm quan trọng là tổ chức phải là pháp nhân doanh nghiệp và bất động sản nên được quản lý một cách tổng thể để hỗ trợ sứ mệnh của công ty. Đây là một hoạt động quản lý chiến lược liên kết chiến lược bất động sản với chiến lược kinh doanh. Nó có nhiều kích thước, quan điểm, công cụ và lĩnh vực hoạt động khác nhau giữa những người khác cần được xem xét một cách tổng thể thay vì chia nhỏ cách tiếp cận của quản lý bất động sản truyền thống. Thông tin là cơ bản để thành công CREM và nhận thức của người sử dụng tài sản là điều 
không thể thương lượng. Điều này theo đó có thể được sửa đổi dựa trên đơn vị tiền tệ của thảo luận, nghiên cứu và thâm nhập vào chủ đề và lĩnh vực chuyên môn của CREM tại bất kỳ thời điểm nào đúng giờ. Xét về tầm quan trọng của $C R E M$ và việc quản lý nó, điều quan trọng là các tổ chức phải tìm kiếm chuyên môn của các nhà chuyên môn. Những chuyên gia như vậy sẽ phát triển và điều chỉnh chiến lược CRE với một chiến lược kinh doanh của tổ chức để đảm bảo lợi ích tối ưu cho tổ chức doanh nghiệp nói trên. Những chuyên gia này nên hoạt động ở cấp chiến lược hoặc tệ hơn là liên kết với sự quản lý của các tổ chức đó trong việc thực hiện nhiệm vụ của mình. Điều này sẽ đảm bảo rằng giá trị gia tăng của $\mathrm{CREM}$ và sự quản lý của nó thực sự được đánh giá cao trong các phòng họp. Điều này sẽ cũng đảm bảo rằng CREM được đánh giá thích hợp và do đó CRE được phù hợp được sử dụng để nó không còn bị xếp vào vị trí "chi phí kinh doanh"; nhưng rằng chất lượng giá trị gia tăng của nó có thể được thể hiện tốt hơn. Để đạt được những điều này, chuyên gia các hiệp hội cần thu hút các giám đốc điều hành kinh doanh để đánh giá cao vị trí độc đáo của CREM và sự quản lý. Một khi những người trong ban lãnh đạo cấp cao hiểu được thực tế rằng $\mathrm{CREM}$ là cần thiết cho sự thành công của doanh nghiệp, mức độ liên quan của nó sẽ tự động được nhìn thấy trong chiến lược quyết định của các chủ thể công ty. Các lĩnh vực thống trị trong nghiên cứu CREM hiện là chiến lược theo sau là gia tăng giá trị, xây dựng thương hiệu và quản lý khu vực công.

\section{Tài liệu tham khảo}

Bon, R. (1994). Ten principles of corporate real estate management. Facilities, 12(5), 9-10.

Bon, R. (1995). Corporate real estate management practices in Europe and the United State: 1993 and 1994. Facilities, 13(7), 1-13.

Hartmann, S., Linneman, P., Pfnuer, A., \& Siperstein, B. (2009). Realising the value of corporate real estate management. Wharton Real Estate Review, 13(1), 21-33.

Hartmann, S., Linneman, P., Pfnür, A., Moy, D., \& Siperstein, B. (2010). Responsibility for and performance of corporate real estate functions. Journal of Corporate Real Estate, 12(1), 7 -25

Jones, K., \& White, A. D. (2012). RICS public sector asset management guidelines. London, UK: RICS.

Krumm, P. J. M. M. (2001). History of real estate management from a corporate perspective. Facilities, 19(7/8), 276-286.

Lambert, S., \& Poteete, J. (1997). Managing global real estate, international development research foundation. Atlanta, GA.

Lopes, J. L. R. (1996). Corporate real estate management features. Facilities, 14(7/8), 6-11.

Lopes, J. L. R. (1997). A meta-model for corporate real estate management. Facilities, 15(1/2), 2228.

Lopes, J. L. R. (1999). An investigation into the main information dimensions of corporate real estate management. Construction Informatics Digital Library. Retrieved April 9, 2013, from http://itc.scix.net/paper x78- 1999-2517

Luokko, A. (2004). Competitive advantage from operational corporate real estate disposals. International Journal of Strategic Property Management, 8(1), 11-24.

Mole, T., \& Taylor, F. (1992). 'Facility Management: Evolution or Revolution' III Facility Management: Research Directions. London, UK: Royal Institution of Chartered Surveyors.

Musa, M. F., \& Baharum, Z. A. (2012). Corporate Real Estate (CRE): Public institution of higher 
learning in Malaysia. Procedia - Social and Behavioral Sciences, 36, 273-279.

Njungbwen, E., \& Udo, G. O. (2010). Benefit of corporate real estate management to higher education institutions. Department of Estate Management, University of Uyo, Nigeria.

Omar, A., \& Heywood, C. (2010). Corporate real estate management's credibility- positioning status, Preliminary investigation of a branding model in practice. Journal of Corporate Real Estate, 12(3), 185-195.

Scarrett, D. (2010). Property asset management (3rd ed.). London, UK: Routledge Press. 